\title{
Evidence and perceptions on GUI test automation - An Exploratory Study
}

\author{
Chahna Polepalle \\ Bleking institute of technology \\ Sweden \\ chpo16@student.bth.se
}

\author{
Ravi Shankar Kondoju \\ AFRY \\ Sweden \\ ravishankar.kondoju@afry.com
}

\author{
Deepika Badampudi \\ Bleking institute of technology \\ Sweden \\ deepika.badampudi@bth.se
}

\begin{abstract}
Context: Software testing requires substantial effort in maintaining GUI test scripts, leading to rework or waste. Therefore, it is important to identify the factors leading to waste in GUI-based automation testing. Objective: To identify testability requirements and factors associated with waste from literature and practice. Methods: We conducted a literature review to identify the state of knowledge and a two-phased interview with practitioners to capture their subjective opinions and gather their views on the state of knowledge. Results: We identified 16 testability requirements and 14 factors associated with waste in GUI test automation. We classified each of them into SUT-related, test-process-related, test-tool-related, human and organizational, environment and cross-cutting. Conclusions: Our results indicate that the generic testability requirements identified in the literature are also valid for GUI automation testing. Our study identifies new findings in addition to the existing literature.
\end{abstract}

\section{KEYWORDS}

GUI test automation, testability, maintenance

\section{ACM Reference Format:}

Chahna Polepalle, Ravi Shankar Kondoju, and Deepika Badampudi. 2022. Evidence and perceptions on GUI test automation - An Exploratory Study. In 15th Innovations in Software Engineering Conference (ISEC 2022), February 24-26, 2022, Gandhinagar, India. ACM, New York, NY, USA, 10 pages. https: //doi.org/10.1145/3511430.3511442

\section{INTRODUCTION}

Interacting with a system under test (SUT) through a GUI is a challenging part of software test automation [10]. The reason is that GUIs are continuously changing during development breaking their corresponding test scripts thereby, causing a hindrance to test automation [15]. The GUI test scripts are to be frequently maintained to ensure they are compliant and can be executed on a new version of the SUT [2]. As a result, implementing the automation solutions for GUI level testing is problematic as it is costly and a tedious activity in practice [20], [16]. Their presence varies across different systems. For instance, traditionally, GUIs were rarely available in telecommunication products directly unless it is especially required

Permission to make digital or hard copies of part or all of this work for personal or classroom use is granted without fee provided that copies are not made or distributed for profit or commercial advantage and that copies bear this notice and the full citation on the first page. Copyrights for third-party components of this work must be honored For all other uses, contact the owner/author(s).

ISEC 2022, February 24-26, 2022, Gandhinagar, India

(C) 2022 Copyright held by the owner/author(s)

ACM ISBN 978-1-4503-9618-9/22/02.

https://doi.org/10.1145/3511430.3511442
[4].

A large Swedish telecommunication organization identified the need to investigate the GUI test automation for two subsystems with a newly developed GUI. The goal was to systematize the GUI test automation process. The GUI-level test automation can be systematized when it is established at the right time and with a suitable approach [13], [17]. Thus, deciding when to automate and how to make automation easier is a challenging question asked frequently [13][35][33], [34]. To achieve the latter, the software can be improved in terms of testability through identification of testability requirements [25]. These requirements ensure effective and efficient automation of GUI testing [12, 33]. As a substantial amount of effort is required before one begins automation, industry practitioners are keen on exploring answers to such questions $[11,33]$ including the local champion at our case organization.

Moreover, as GUIs are known for their frequent modifications, they cause unnecessary rework in maintaining the test scripts termed as "waste" [2]. Thereby, it is crucial to also identify factors affecting the unnecessary maintenance of GUI automation test cases which often results in waste. If such answers are known and followed, practitioners can control the test effort and may find it to be less tedious to perform GUI test automation [11, 12].

The need for investigation identified by the local champion and the importance of the research topics led to the identification of related work. Different studies have reported on decision support approaches regarding when to begin automation ([34],[14]), testability requirements [25] and factors affecting waste of automated testing [7]. Though literature exists regarding such decisions, it is important to validate if the results related to overall testing apply to GUI test automation and find additional context-specific findings. It is worth investigating as these variables might differ for GUI test automation as its interface changes frequently, and the GUI itself must be tested to verify its conformance to the GUI specifications $[9,22]$. Existing research specific to GUI automation provides testability requirements and maintenance factors. However, this evidence has not been aggregated and interpreted so far. Therefore, to address the research gap, this study aims to aggregate evidence on test automation and validate its applicability in GUI automation in a telecom company. In particular, evidence on the testability requirements which make GUI automation testing effective and efficient and the factors that lead to waste are considered in this study. The identified answers will support the practitioners in making informed and favourable GUI automation decisions.

We implemented the evidence-based software engineering (EBSE) [19] process in the case organization, where we investigated two subsystems relatively new to the field of GUI development. We conducted interviews within the two subsystems, which follow a 
similar approach for GUI based automation testing while varying mainly in terms of the size of the GUI developed. We conducted a two-phased interview to collect the practitioners' subjective opinions and gather their opinions on the evidence collected from the literature. We adopted the Bayesian synthesis method [5] which consists of collecting practitioners' prior opinions and then collecting revised opinions in the light of evidence from the literature.

The paper is structured as follows. Section 2 contains related work. In Section 3, we present an outline of the research methodology. A detailed description of the literature review and the interview results are provided in Sections 4 and 5. Discussions are reported in Section 6.

\section{BACKGROUND AND RELATED WORK}

In the following sections, we present an overview of the related studies.

Testability requirements: Testability makes the software product easier to test in an automated manner either by making the design of the tests easier or testing more efficiently [25]. Though researchers and practitioners have assumptions on what makes a software testable, a validated set of testability requirements has not yet been defined [25]. Pettichord [26] cites examples of testability requirements such as assertions and resource monitoring from his own experience. On a similar note, Patwa et al. [25] also mentions a few examples of testability requirements related to GUI test automation, like proper naming standards for user interface elements and tools for recognizing custom controls. As part of future work, the paper identifies the need for further empirical studies to establish testability engineering as a part of software engineering research. A study at Microsoft [36] finds that the testability of the SUT must be considered with the design and system architecture to analyze how it affects the testing effort and overall testing process. Karhu et al. [18] also view testability of the software as a large concern because poor code can make the testing process unreliable. A case study performed by Liebel et al. [20] reports a lack of testability in the software product as an issue that made the automation testing difficult. However, the paper did not mention any testability requirements or a detailed description of the issues. Xie et al. [38] suggest strategic usage of assertions and creating a minimal number of test cases as examples of testability requirements for automation. They further mention that decisions taken by designers impact not only usability but also testability. As per Alanen et al. [1], it is crucial to involve both testers and developers in understanding testability requirements to enhance software testability. Furthermore, they must promote testability earlier in the development phase and make the SUT easier to test by providing guidelines that aid testability $[1,25,26]$.

Factors affecting waste: Maintaining test cases is generally viewed as an expensive and time-consuming process by software developers [23]. A study at Accenture revealed that even simple changes to GUIs lead to $30 \%$ to $70 \%$ modifications to the test scripts making $74 \%$ of the test cases unusable during GUI regression testing [15]. Valid empirical data on maintenance is limited, especially when it comes to GUI test automation [2]. In their systematic literature review (SLR) of merits and drawbacks of automated testing, Rafi et al. [28] reported only four papers that describe the difficulties associated with the maintenance of automation test cases. Out of which, only one study identified factors that affect the maintenance in the form of theoretical cost models [7]. Similarly, some studies, $[3,15,18]$ stated several factors, both technical (standardized technological infrastructure and frequent changes to the underlying technology) and context-dependent (undocumented architecture and poorly structured testware), which impact maintenance of automated testing. Karhu et al. [18] performed a case study where they observed the factors that influenced the use of software testing automation, like considering maintenance costs as well as human factors. Their observations are supported by Berner et al. [7] who further propose that the design of the test suite architecture is also an important factor that is commonly overlooked. In addition, the absence of proper testware documentation and lack of guidelines creating reusable and maintainable tests are additional factors affecting waste [2, 7, 18]. Moreover, Alègroth et al. [2] reported thirteen factors influencing maintenance and their impact on automated GUI based testing, represented by Visual GUI testing (VGT). Furthermore, companies generally abandon automated testing even after substantial investment due to wrong expectations while implementing test automation $[2,7,18]$.

Research gap: There exists evidence regarding testability requirements and factors affecting waste for automation in general and a few of them for GUI test automation (some testability requirements in [26], and maintenance factors in [2]) as well. There is no study aggregating evidence on the testability requirements influencing software test automation. The SLR proposed by Rafi et al. [28] reports only four papers discussing maintenance associated with automation test cases but not specific to GUI test automation. A multi-vocal literature review [13] proposed 15 factors that support decision-making on whether and what to automate in software testing. It does not discuss the testability and waste. Garousi et al. [13] identify the need to validate the research evidence in an industrial scenario for a specific context. Not all variables influencing GUI test automation are likely suitable for every industry. For instance, even though GUI testing is widely practised, its availability varies on different platforms. GUIs are rarely present in telecommunication products [4]. Thus, the identified evidence might not be applicable in such a context. It is worth investigating as these variables may be different for GUI test automation in such a context owing to their dynamic nature. Moreover, GUI itself must be tested to verify its conformance to the specifications $[9,12,22]$. As identified in some studies, it is essential to identify additional variables and understand how such software test automation decisions and maintenance factors are affected in a specific context [2,13]. There has been a steady increase in the number of articles related to GUIs, mainly contributing towards improved testing techniques, tools, and testing paradigms $[6,16]$. Nonetheless, only $13.23 \%$ of the articles have been published in collaboration with industry while $73.52 \%$ of the papers are solely published by the academia [6]. There has been a lack of articles investigating the opinions of practitioners towards GUI test automation and also researchers about their opinions on the current state-of-the-knowledge and on possible future research directions, $[6,16]$.

Overall, the lack of context-specific and validated evidence is the main motivation for this study. This study is motivated by the industrial need coupled with the research gap as identified from 
the literature. Furthermore, this research validates the empirical evidence in an industrial scenario and contributes to the body of literature.

\section{RESEARCH METHOD}

The overall goal of the research is that practitioners involved in GUI test automation will be able to make informed decisions regarding GUI automation. An expert panel discussion was conducted with the local champion which lead to the formation of the following research questions:

RQ1. What are the testability requirements for performing GUI test automation?

RQ2. What are the factors that lead to waste in GUI test automation?

\subsection{Literature review design}

We conducted a literature review using the snowballing approach by following the guidelines proposed by Wohlin [37]. Our intention was to find the maximum number of available sources addressing the research questions in a systematic manner. The purpose of snowballing was to find evidence on testability requirements and factors associated with waste and not on overall related work on GUI automation.

Start set for snowballing: We identified keywords based on the research questions. Then we formulated relevant search strings by combining the keywords. The search strings were, (GUI testing OR GUI test automation), agile AND (GUI testing or GUI test automation), GUI test automation AND testability requirements, "testability requirements" AND (GUI testing OR GUI test automation), (GUI testing OR GUI test automation ) AND (maintenance OR maintenance costs). For each search string, the first ten results of Google Scholar were examined by checking their abstract and title for relevance to generate a tentative start set. Next, the full text of each paper from the tentative set was reviewed. Nine studies were obtained, forming our final start set.

Snowballing: We conducted backward and forward snowballing to find sources of papers in the chosen research area. It is an iterative process that spans until no new studies are found. Ten additional papers were identified, making it 19 in total.

\subsection{Interviews}

Interviews are conducted for capturing the perceptions of the practitioners to obtain qualitative data. Overall all 18 practitioners involved in the testing were approached, of which a few were unavailable for participation. Thus, we interviewed a total of 12 participants from the two subsystems. Moreover, we interviewed at least one practitioner per role per subsystem, thereby capturing opinions from different roles.

Interview design: We have adopted a semi-structured interview strategy [30] for all the interviews. As the study is exploratory, a semi-structured interview was suitable to gather in-depth and better-quality information as it allows to ask to follow up questions when interesting points were raised by the interviewee [29].

Formulation of interview questionnaire and analysis: A semistructured questionnaire was designed based on literature review and case organization's process documents. The interviews were recorded after obtaining informed consent and transcribed word to word. We used thematic analysis [8] to analyze the transcribed data.

Analysis: We followed the six steps thematic analysis framework proposed by Braun and Clark [8] to analyze qualitative data from interviews and literature results. As our study focuses on capturing the practitioners' opinions, we selected the generic thematicanalysis approach. We started the analysis by becoming familiar with the data, where we thoroughly read the primary studies and interview transcripts. Then we generated a list of initial codes by highlighting data and assigning "codes". The codes were then classified into themes conceptually similarity. We also reviewed and defined the generated themes. The entire analysis process was conducted by the first two authors and reviewed by the third author.

\subsection{Overview of cases and GUI test automation}

The characteristics of each case are mentioned below.

Case 1 description at subsystem 1: Subsystem 1 is composed of entities that offer a business configuration (enabling/disabling services) to the end-users and are heavily dependent on the GUI. Their GUI sets up specifications of the entities which are required to create product offerings. They develop a large amount of GUI code $(111,236 \mathrm{LOC})$ and write an equivalent number of GUI test cases $(\sim 850)$.

Case 2 description at subsystem 2: Subsystem 2 composes of several entities that offer services for executing the business configuration produced by subsystem 1 . Their GUI is flexible and is used to set up the configuration for the services offered. They are not heavily dependent on the GUI, and hence the GUI produced is smaller (57,653 LOC and 250 test cases) in comparison to subsystem 1.

Overview of GUI test automation at the case organisation: Within both the subsystems, the GUI test automation is applied at Unit, Integration and Black box levels. The unit tests are used to verify the input and output of independent GUI code sections and any modifications made to the Document Object Model (DOM). In the integration testing, the functional coverage of each business use case is tested. It aims to test components such as widgets, regions or an entire application and the flows in an application or a page. In black-box testing, it is ensured that the connections between the GUI, server and other dependencies work in all circumstances.

\section{LITERATURE REVIEW RESULTS}

A literature review was conducted to identify evidence for addressing our research questions. The evidence collected from the literature review is provided to the interviewees in Phase 2 of the interview. Based on our literature review, we present the criteria for testability requirements and several factors associated with unnecessary maintenance of automation testing. Generic findings related to automation which are not limited to GUI test automation, are extracted from the research studies. The findings related to each research question were further divided into categories using thematic analysis. Firstly, the codes were organized as per our research questions, i.e. testability requirements and factors associated with waste. Next, they were placed into categories that we found to best explain the codes. The categories include test-related, test-tool related, SUT-related, human and organizational, and cross-cutting. Each category is described as follows - 
Test-process related: This theme refers to characteristics of test cases or test suites and relevant testing activities.

Test-tool related: Findings related to the quality, support and selection of appropriate GUI test automation tools are included in this theme.

SUT-related: SUT related category explains the properties of the system under test (SUT).

Human and organizational: Findings related to humans and organization, such as availability of resources and characteristics of developers, are in theme.

Environment: This theme is related to the test environment.

Cross-cutting: On analyzing the data, we observed that certain codes have an implicit relationship with other categories, thereby making them applicable in the context of more than one category. We named such a category cross-cutting.

4.0.1 Testability requirements. Testability is one of the criteria to be considered before one begins automation [32] and [31]. Ten testability requirements are identified shown in Table 1 . As described earlier, testability requirements are not related to SUT alone but are affected by the automation tool and the characteristics of automation tests. Hence, testability requirements are divided into four categories using thematic analysis. Use of unique names for different windows and interface controls and support for custom controls are the two testability requirements identified as specific to GUI test automation. Use of effective event log storage management is identified as the most important testability requirement based on the frequency of occurrence in literature. Having effective log storage management is the most reported testability requirement. In the interviews, we contextualized and validated if all these testability requirements are valid for GUI automation.

Table 1: Evidence on testability requirements

\begin{tabular}{|l|l|}
\hline Testability requirements & References \\
\hline Test process & \\
\hline Establish test scripting standards & {$[11][7]$} \\
\hline $\begin{array}{l}\text { Identifying and converting common steps into } \\
\text { functions }\end{array}$ & {$[11][7]$} \\
\hline Clean-up between test runs & {$[11]$} \\
\hline SUT & \\
\hline Effective event log storage management & {$[11][26]$} \\
\hline Usage of diagnostic techniques & {$[7][1][24]$} \\
\hline $\begin{array}{l}\text { Use of unique names for different windows and } \\
\text { interface controls }\end{array}$ & {$[26][7][1][24][1][24]$} \\
\hline Usage of monitoring techniques & {$[26][7][1][24]$} \\
\hline Usage of fault injection techniques & {$[26][1][24]$} \\
\hline Test-tool related & {$[7][26][17][25]$} \\
\hline Support for custom controls & {$[26][7]$} \\
\hline Environment & \\
\hline Automatic installation and configuration & {$[26$} \\
\hline
\end{tabular}

Table 2: Evidence on factors related to waste

\begin{tabular}{|l|l|}
\hline Factors associated with waste & References \\
\hline Test-process & \\
\hline Poor variable names and script logic & {$[7][2]$} \\
\hline Inconsistent test cases & {$[7][2]$} \\
\hline Broken test scripts & {$[7][27]$} \\
\hline Long test cases & {$[2]$} \\
\hline Loops and branches & {$[2]$} \\
\hline Lack of testing of test code & {$[7][11]$} \\
\hline Lack of structured test suite architecture & {$[2][7]$} \\
\hline SUT & \\
\hline Number of GUI interaction components & {$[2]$} \\
\hline Human and organizational & \\
\hline Inadequate knowledge and experience & {$[2][7][18][28][15][21]$} \\
\hline Test-tool related & \\
\hline Lack of tool support for automation & {$[2][18]$} \\
\hline Environment & \\
\hline Inconsistent simulator & {$[2][7]$} \\
\hline Cross-cutting & \\
\hline Constant technological changes & {$[18]$} \\
\hline
\end{tabular}

4.0.2 Factors associated with waste. 12 factors which negatively affect the maintenance of GUI based automation testing are identified from the literature and presented in Table 2. Most of these factors are found in the context of GUI automation testing. These factors were divided into 6 categories using thematic analysis. Knowledge and experience was observed to be the most reported factor influencing maintenance of test scripts.

\section{INTERVIEW RESULTS}

The interviews aim to collect practitioners' perspectives on GUI automation, validate the evidence from existing research, and identify new findings that are not reported in the literature.

\subsection{Summary of interviews}

In order to obtain relevant qualitative data from the two subsystems within the industry, a total of 12 interviews were conducted. A summary of the interview participants is presented in Table 3. To elicit unbiased opinions, we first asked the interviewees about their opinions without discussing any findings from the literature (Phase 1). In the later part of the interview (Phase 2), we asked about their opinion on the findings from the literature. Section 5.2 presents the results that were acquired in Phase 1 of the 12 interviews.

\subsection{Phase 1 interview results for testability requirements}

In the first phase of the interview, we asked for unbiased opinions of the practitioners regarding testability. This phase refers to collecting prior opinions in Bayesian synthesis [5]. The practitioners mentioned some of the factors that were reported in the 
Table 3: Summary of interview participants.

\begin{tabular}{|c|c|c|c|}
\hline Interviewee & Subsystems & Experience & Role \\
\hline Interviewee 1 & Subsystem 1 & 7 years & Test architect \\
\hline Interviewee 2 & Subsystem 2 & 2 years & Developer/Tester \\
\hline Interviewee 3 & Subsystem 1 & 11 years & Test lead \\
\hline Interviewee 4 & Subsystem 2 & 7 years & Product owner \\
\hline Interviewee 5 & Subsystem 2 & 5 years & Design lead \\
\hline Interviewee 6 & Subsystem 1 & 9 years & Test lead \\
\hline Interviewee 7 & Subsystem 1 & 3.5 years & Product owner \\
\hline Interviewee 8 & Subsystem 2 & 22 years & Test architect \\
\hline Interviewee 9 & Subsystem 1 & 2.5 years & Developer/Tester \\
\hline Interviewee 10 & Subsystem 1 & 7 years & Design lead \\
\hline Interviewee 11 & Subsystem 2 & 12 years & Test architect \\
\hline Interviewee 12 & Subsystem 2 & 6 years & Test lead \\
\hline
\end{tabular}

literature. In addition, practitioners mentioned new requirements that were not reported in existing literature, such as performing manual testing prior to automation testing, Defining clear product requirements, using GUI development framework, Synchronous behaviour, and using a minimal number of anonymous functions and Automation tool documentation/support.

Following are the descriptions of the testability requirements mentioned in Phase 1 -

\section{Test process related}

T1: Establish test scripting standards. From practitioners perspective, coding standards improve the quality of test scripts which in turn facilitates test automation. The two subsystems in the case organization have rules that should be followed when writing test scripts that are available as a wiki page. In addition, Sonarqube ${ }^{1}$ is used to configure the required rules. One of the interviewees mentioned that "its[Sonarqube] a very good tool in making sure you write decent code". However, a test architect mentions that using Sonar is not enough, "Sonar doesn't help in creating robust and maintainable test cases as it is a static code analysis tool". Thus, it is essential to create guidelines and a practical approach for writing robust test scripts. A particular approach to adhere to the coding standards are important as one of the interviewee mentions that "there is always a risk of doing too much of that [formalizing] but it wont be followed. It sounds good on paper but having too much process and stuff only makes people skip it". Automating the process may eliminate the need to have heavy-weight processes to ensure compliance to coding standards.

T2: Identifying and converting common steps into functions. This is perceived as important in GUI testing as certain UI elements can be reused as mentioned by of the interviewee - "While writing selenide tests, there are class names or widgets that can be common for many pages. So we suggest our designers to have these widgets as a common one. We will write one method for the widget and we can take the same thing for all the test cases".

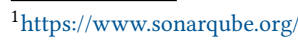

T3: Clean-up between test runs. In GUI testing frameworks, the test cases are dependent on each other and modifications in one test case might affect the execution of subsequent test cases. For instance, one interviewee mentions,"In Selenide, sometimes when we have one failing test case, the following test cases will also fail as they can't find where to click, or they click in the wrong places since the previous test case dint clean up after it. That's a problem in all GUI test frameworks. I wanted to able to reset the data we had between runs but, it isn't possible in Selenide as it is written now". Moreover, manually resetting the data between test runs is problematic since every step needs to be repeated and executed again. Thus, it is important to automatically clear or reset data between test cases after each run.

T4: Perform manual testing prior to automation testing. Owning to the nature of the GUIs, which change frequently, this testability requirement provides an opportunity to understand the behaviour of the GUI and implement the same for generating automation test cases. Moreover, interviewees perceive that such a strategy enables detecting and resolving errors that might result in failing the GUI automation test case quite early in the development cycle. In addition, testing manually will save time in the long run as the product will be more stable and will contain minimal errors due to early testing. A test lead mentions that "Since it is GUI we cannot always do automation first, we start testing manually and then automation".

\section{SUT related}

T5: Effective event log storage management. Interviewees perceive is important to verify if the logs contain enough information to understand if the problem is in production or test code. One of the test lead mentions, "I think it could be clearer messages. If there is a configuration file error, then it should say what configuration file and in best case, what place in the configuration". For GUI test automation, it is advantageous to have snapshots indicating the parts of the GUI where an error occurred. The visuals coupled with console logs increases the effectiveness of the logs and helps testers identify the error quickly. Thus, the effectiveness of the logs can always be improved depending on practitioners need.

T6: Usage of diagnostic techniques. Assertions are used to perform such diagnosis in testing."For every step we do, we assert to check if it has correct values". Moreover, open-source tools are available, which makes the diagnosis easier. "We are using chrome dev tools, and without the tool, we wouldn't be able to debug GUI tests. You can always have more tests, but a tool still makes life easier". Such diagnosis is beneficial for all types of automation tests, particularly for GUI automation testing. Several frameworks and assertion libraries exist for developing GUI test cases to diagnose if the GUI elements return the expected behaviour.

T7: Use of unique names for different windows and interface controls. Use of unique class names ensures that the automation tool can identify the right component when multiple components with the same identifier exist. For instance, if certain GUI components use the same class name, the complexity increases while identifying the required component for testing. This is because the same class name is assigned in several places and tests usually fail for detecting incorrect element as mentioned by one of the interviewee, "Its hard 
to find the element that you need on the page if you have the same class name in a lot of places". While another interviewee adds, "Using unique names reduces complexity a lot and I believe that tests become more robust".

T8: Define clear product requirements. As mentioned by a testarchitect "To be testable could imply that the requirements are extremely clear and not fluffy so we know what we are supposed to verify, that is one kind of testability". A similar experience has been presented by a test-lead "You don't want to have a requirement that is a one-liner. You need to know if you are able to develop tests that you can actually check that it is true or not". Product requirements need to be clear to ensure that the system is testable and verified efficiently by the tests.

T9: Use of GUI development framework. The framework used for developing the GUI should be testable. A test architect mentions that "Selenium isn't that compatible with the GUI framework developed in the company and hence trying to push our tests down to its integration tests. We seek more testing at that level and then keep basic end to end flows on a black box level to minimize the incompatibility". For higher testing levels, such as an end to end testing in black-box tests, external automation tools might be required for testing. To ensure compatibility of the development framework with the automation tool, the GUI and its underlying framework should be developed with testability in mind.

T10: Synchronous behaviour. Synchronous behaviour is a mechanism to know if the system is in a ready state after the accomplishment of all the asynchronous events. Once the events are processed, the system must respond that it is ready for automation without manually checking its behaviour. One of the interviewees mentions, "We want synchronous behavior, where all the asynchronous events have been propagated to the system. We need a way to know if the system is ready. For example, the configuration that has been sent is processed already, so that I can run my test. And that is extremely difficult to achieve". The preparation and ready state of the system ensures the GUI tests run without any timeout issues.

T11: Use of a minimal number of anonymous function. The use of a minimum number of anonymous functions in GUI code is beneficial as they make test automation less difficult. The use of these functions can be reduced by using callbacks to lift the call back on a named declared function. It is easy to test this function or mock it out rather than testing a large function to test a small anonymous inline function for its behaviour. A developer mentions that "I try to minimize the use of anonymous functions for instance since they can be really hard to test".

Test-tool related

T12: Automation tool documentation/support. To perform test automation in an efficient and an accurate way, it is necessary to refer to the documentation of the tool. This ensures that the full capability of the automation tool and can be referred to and implemented, making automation testing more organized and easy. A test lead claims that "I think the best part of the tool is that it is well documented and it's easy to find others that uses it so you can get help when you get stuck in it. Moreover, access to user groups and forums holding discussions on the tool benefits the tester to identify a quick solution in case of a problem. This is important for GUI automation tools to work with the system being tested.

\subsection{Phase 2 interview results for testability requirements}

EBSE recommends validating the research-based evidence obtained from literature by integrating the evidence with practitioners' opinions and experiences. This phase refers to collecting revised opinions in Bayesian synthesis [5]. In this phase, all findings are discussed. Even those that were mentioned in Phase 1 as a subset of interviewees only mentioned them. This process is followed to get a collective opinion on the findings by interviewees. Following are the opinions of practitioners on the literature results that they did not mention in Phase 1 -

SUT related Usage of monitoring and fault injection techniques were not mentioned by any of the interviewees in Phase 1. Upon asking for their opinions, we got the following responses -

T13: Usage of monitoring techniques. One of the interviewees mentioned -" We have traced. From GUI black-box perspective you can activate trace and follow what's happening". However, it is not as detailed as pointed out by another interviewee - "We have this trace files that typically use to see how far in the flow you have come, For GUI we don't have the same, we only have logs, we don't have this trace mechanism to see flow inside the system."

T14: Usage of fault injection techniques. Interviewees' perceive that the fault scenarios cannot be simulated in black box level while it is possible in integration levels. One of the interviewees mentions "There is no way to fake real errors in a black box system. We can't fake that, but we are mocking that in previous test cases like in unit and integration tests to simulate those fault scenarios".

Test-tool related

T15: Support for custom controls. In the current subsystems, the tools are configurable to be able to detect custom controls. "It will show up in DOM as anything else, so if you write test cases for it, if you say to look for this button, it will find it". Moreover, plugins can be developed for the automation tools to improve its capability in recognizing different types of GUI components.

\section{Environment}

T16: Automatic installation and configuration. This ensures that the software being tested is installed automatically using install scripts on several machines with varying configurations. Provision of such install scripts enables the practitioners to focus mainly on the development of automation test scripts and saves time by not configuring software manually. "This is very important otherwise development would be standing still if we dint have automation and config automatically".

\subsection{Synthesis of evidence and opinions on testability requirements}

Figure 1 shows the frequencies of interviewees mentioned testability requirements and the frequency of papers reporting. For example, if $2 / 12$ interviewees mentioned the factor, the frequency is $17 \%$. Similarly, if $2 / 7$ primary studies report a factor, then the frequency is $29 \%$. We mainly asked the interviewees their opinion on 


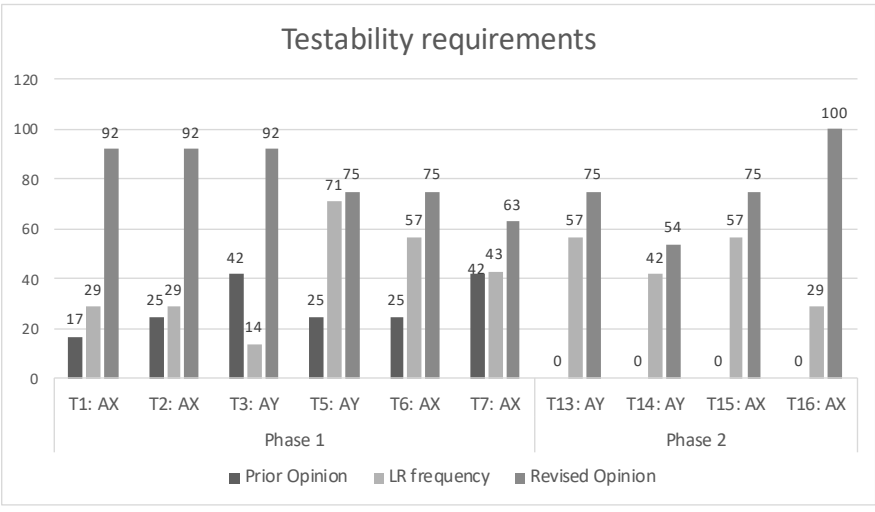

Figure 1: Synthesis of evidence and opinions on testability requirements

literature results and whether they followed the testability requirements in their automation process. In Figure 1, each TR is suffixed with AX or AY, A: denotes if they agree with the results and if they follow (X) or partially follow (Y) in practice. In summary, a small set of interviewees accounted for some testability requirements, which significantly increased after the results from the literature were discussed. This shows that the literature results allowed the practitioners to become more consensual and aware of the testability requirements. Some of the requirements were not mentioned in Phase 1 as they are already followed and are part of practitioners implicit knowledge such as T15:AX and T16:AX. In other cases, it is not always feasible to fully consider the testability requirements such as T13:AY and T14:AY. Interestingly, even when practitioners mention some of the requirements in Phase 1, they are not fully followed, such as T3:AY and T5:AY, which are related to cleaning between test runs and effective log storage. These requirements are identified as improvement areas. Some interviewees were not sure about some requirements and did not prefer to comment or were unsure hence, the revised opinion is not always $100 \%$.

\subsection{Phase 1 interview results for factors associated with waste}

Similar testability requirements, the interviewees mentioned some of the factors related to waste that was also reported in the literature. In addition, the interviewees mentioned two new factors (Ineffective sleep functions and attrition of GUI developers).

Following are the descriptions of the factors associated with waste mentioned in Phase 1 -

\section{Test-process related}

W1: Broken test scripts. It is perceived important as one of the interviewees mentioned - "If you are changing code, you are breaking the old functionality. Then you should update it as you have made a change. If it's an expected change, the implementation must be done smartly to reduce the effort to update the tests. However, if the fix is highly complex or the test covers a functionality which does not exist anymore, the test should be eliminated from the suite".

W2: Lack of structured of test suite architecture. A poorly structured test suite requires additional effort for maintaining the test scripts.
Previously, one test file was created for each use case in the subsystems. This led to the generation of many test files residing in a directory. Whenever the GUI changed, the test cases broke, and one had to edit each of the affected test files to resolve the issue. Moreover, it is difficult to track the defective file in the large set from a maintenance point of view. "It is difficult to understand the fix and the workaround implemented. All the files that are lying around everywhere. It's a big problem. There was no real structure when coding began". The architectural decisions must be taken after careful analysis and investigation. It is essential to produce standardized guidelines and document the structure of test suite architecture.

W3: Ineffective sleep functions. A developer mentions the issues with sleep functions as "If you assume the page to load in $5 \mathrm{~s}$, it might load in 5ms. This increases execution time. It's important to do a continuous check to find the appropriate time. 1s might work in most of the cases. But, when the computer slows down, it results in blinking test cases". Sleep functions increase the test case execution time and timeout issues resulting in wastage of maintenance effort. To mitigate this problem, it is advisable to develop the test scripts to wait for a GUI event.

\section{SUT related}

W4: High number of GUI interaction components. The number of GUI interaction components can be reduced by reusing GUI components. However, it is not always straightforward as mentioned by a product owner, "All the GUI pages have some general components. But most of the pages also have specific components, and adding these breaks the existing automation test cases. So maintaining GUI automation test cases is a bit hard as GUI should look the same way while having specific things".

\section{Human and organizational}

W5: Inadequate knowledge and experience. The interviewees mentioned problems due to inadequate knowledge and experience - "We didn't have much experience with GUIs as it is new for our company. We assumed that by hiring many young people with good knowledge of the product, they might have good experience in creating GUI automation tests. It turned out to be extremely not true". Practitioners lacking knowledge and experience tend to develop poorly structured GUI frameworks and unstable test scripts. Thus, domain experts should perform GUI test automation as mentioned by one of the interviewees - "We should have taken the time to create basic frameworks and hired someone with great GUI competence to get the basics up. This would have helped us gain knowledge on developing maintainable suites".

W6: Attrition of GUI developers. Knowledge translation will be a tedious process as the person responsible for the specific part in the GUI is unavailable. According to the experience of a design lead, "Someone writes part of code and then quits. No one really owns that part anymore". Thus, maintenance effort aggravates due to the high attrition rate of practitioners working in the industry. Another interviewee mentions, "one guy developed major functionality and later quit the company. It's badly written and nobody takes the responsibility to improve it". Organizations generally recruit consultants to assist in development to cope with time pressure. But their fixed contracts hinder the continuity of GUI development and test 
automation causing additional maintenance problems.

\section{Test-tool related}

W7: Lack of tool support for automation. Using older versions of the automation tool is a liability due to restrictions of the tools' availability towards new programming languages and features. For example, at the case organization, practitioners had issues with the older version of the tool since certain features were not available or not supported by it. However, the fix was already released in the new version." Selenide doesn't support HTML5 drag and drop. There are workarounds but not for the old Selenide version that we are using ". Limitations in the automation tool will hamper test script development and maintenance.

\subsection{Phase 2 interview results for factors associated with waste}

Following are the opinions of practitioners on factors that were not mentioned in Phase 1 -

Test related process

W8: Poor variable names and script logic. A design lead says, "The names affect very much. A continue button is named colour blue. If we change that name, then we need to maintain that in the tests. The colour blue is a bad name. It should better be continue button instead". Moreover, it is advisable to have common class names and widgets used at several places as a single method that can be used to test the common code elements.

W9: Inconsistent test cases. One of the interviewees mentioned that - "If you have a maintenance team trying to execute the tests and then we have a part that changes and if they don't follow the same cycle you will have increased maintenance costs". Implementing a continuous integration framework that runs the tests every time a code is pushed or committed is advantageous. If the tests pass, the tool must change to green if not red. Thus, the test cases must be run consistently several times a day to reduce the maintenance problems of the test cases.

W10: Long test cases. One of the interviewees mentioned - "I' $m$ a firm believer that all code should be short. It should fit on the screen so you can see it without scrolling". If there are a large number of nested elements, it leads to long test cases that can be hard to read and find what to test. However, it is not always possible, as pointed out by one of the interviewees - "We want to keep them short but, selenium and integration tests are long as they need to go through the entire GUI. But others like unit tests are very short".

W11: Lack of testing of test code. Problems with test code was mentioned - "We found one test case last week where we thought the test tested correctly but it didn't. It passed all the time. So it's important to test the test code". However, few practitioners disagreed with this factor and saw it as a wastage of effort. A consensus was agreed that the test cases must be tested superficially. One possible solution is to run through the test case manually and verify if it works. "Run the test case manually. If there is a statement in the code, then you need to make sure you have tested it manually".

W12: Loops and branches. The interviewees mentioned that they do not use loops and branches when writing test cases for both the

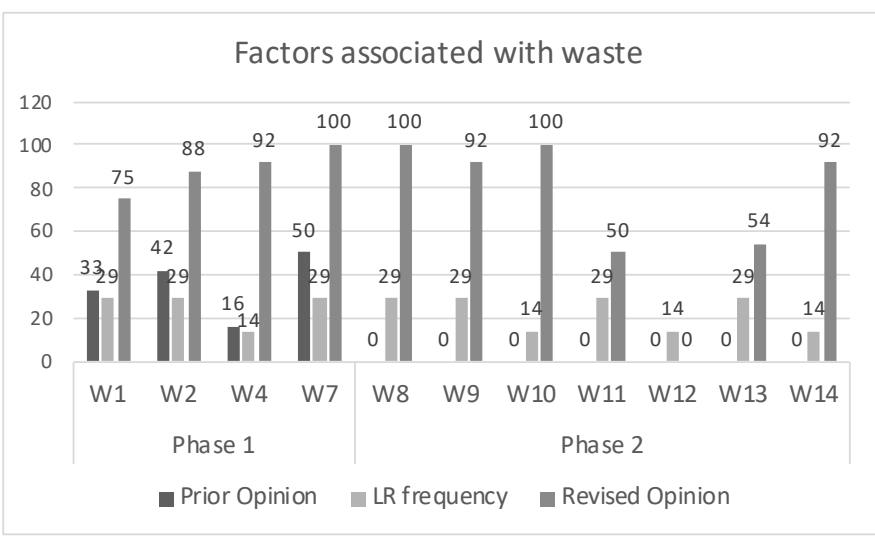

Figure 2: Synthesis of evidence and opinions on waste

subsystems.

Environment

W13: Inconsistent simulator. Simulators are shared between the two subsystems in case of organizations emulate certain parts of their environment. Thus, if there is any update or modification in the original environment, the same should be done in the simulated environment as well. "It needs to work with whatever functionalities required of it, so its maintenance cost". This demands additional effort for updating the relevant test scripts." As we are using different simulators when there are any changes in the actual code, we need to update as well".

Cross-cutting

W14: Constant technological changes. A product owner claims "If we switch from Ymer to angular $7 S$ it will affect maintenance because we need to update a lot of GUI tests". If the technology change is introduced in the middle of an ongoing project, it will need additional effort to adapt to the change. For instance, one of the interviewees mentions, "When the GUI was introduced, Java designs were used, but later we switched to favaScript (FS). As not many had JS competence, the language in itself has been a challenge. Writing corresponding automation tests is a bigger challenge".

\subsection{Synthesis of evidence and opinions on waste}

As in Figure 2, only a few factors were mentioned by interviewees in Phase 1. Similar to Figure 1, the frequencies of interviewees mentioning a factor in Phase 2 increased significantly compared to Phase 1. In summary, some of the factors were not mentioned as they are not exposed to certain waste factors, such as the use of loops as branches (W12) as they are not widely used. In addition, some of the interviews had mixed views on factors such as testing test code (W11). While some factors were acknowledged to be important, however, it was not always possible to mitigate the factor, for example, writing short test cases (W10). 


\section{DISCUSSIONS}

The 16 testability requirements are classified into four categories, namely SUT-related, test-process related, test-tool related and environment. 10 testability requirements are identified from literature base and 12 are obtained from prior subjective opinions of the practitioners as described in Section 5.2. Use of unique class names [26] and support for custom controls [26] are the two variables found in literature to be specific to GUI automation testing while the remaining 8 testability requirements were found in the context of test automation in general. The general category testability requirements were validated and applicable for making GUI based automation testing easier. The category SUT related is found to be the most important category while considering testability requirements as it consists of a maximum number of testability requirements. This is also mentioned in literature [7]. Furthermore, subjective opinions of the practitioners helped in identifying six new variables define clear product requirements, use of GUI development framework, synchronous behavior, use of minimal number of anonymous functions, perform manual testing prior automation and automation tool documentation/support.

The 14 factors associated with waste in GUI test automation are also divided into six categories. Twelve variables were identified from the literature, and then eight were obtained from subjective opinions of the practitioners as described in Section 5.5. Knowledge and experience is identified as a primary factor associated with unnecessary maintenance from literature and also has high validity in the context. Out of the 14 final sets of variables, loops and branches is perceived as not applicable in the context under study. However, $92 \%$ of the practitioners feel that it affects the maintenance of GUI test scripts but is not valid in the context as loops and branches are not used for writing test cases. Most of the factors were identified from the literature as applicable to GUI based automation testing as well as software testing in general [2]. The category test-process related consists of a maximum number of factors associated with waste indicating its higher importance over other categories. Two new factors applicable to GUI test automation were identified from the subjective opinions of the industry practitioners. Sleep functions are used for synchronization of GUI test scenarios with the system being tested. Invalid sleep rages cause timeout issues and increase the total script execution time, making maintenance of GUI test scripts difficult. High attrition rate of GUI developers affects maintenance cycles, especially because the telecommunication industry is relatively new to GUI development and testing and knowledge translation is a tedious process.

\section{VALIDITY THREATS}

One of the threats might be a misinterpretation of the interview questions. A pilot interview was conducted with an industry practitioner, a test architect at the case organization. The pilot interview helped us to refine our questions in the company's terminology. Our interviews were limited to our case organization. This threat is plausible. However, the aim was to contextualize the literature evidence into a specific case, i.e., GUI testing in telecom applications. Therefore, we do not aim to generalize the results to other contexts.

\section{CONCLUSION}

Software test automation proves to be beneficial and ensures success only when implemented carefully [12]. It is important to identify the testability requirements which must be met to make automation effective [12] and [25]. GUIs are known for changing continuously during development and breaking their corresponding test scripts, hindering test automation [15]. Maintaining such test scripts is an expensive and cumbersome process, so identifying the major factors leading to such waste becomes essential.

The testability requirements identified in this study should be considered when planning GUI automation testing. Furthermore, the system not developed to meet testability requirements increases the overall unnecessary maintenance effort (waste) of test scripts, particularly in GUI based automation testing. Hence, testability level is an important criterion for automating and has a significant influence on shortening the maintenance cycles. We translated research-based evidence into practice by combining the subjective opinions of the practitioners with evidence. Practitioners can use the produced context-specific results to make more informed decisions regarding GUI automation testing by relying on evidence and being cautious about maintenance factors.

\section{REFERENCES}

[1] Jack Alanen and Louis Y Ungar. 2011. Comparing software design for testability to hardware DFT and BIST. In AUTOTESTCON, 2011 IEEE. IEEE, 272-278.

[2] Emil Alégroth, Robert Feldt, and Pirjo Kolström. 2016. Maintenance of automated test suites in industry: An empirical study on Visual GUI Testing. Information and Software Technology 73 (2016), 66-80.

[3] Yasaman Amannejad, Vahid Garousi, Rob Irving, and Zahra Sahaf. 2014. A searchbased approach for cost-effective software test automation decision support and an industrial case study. In Software Testing, Verification and Validation Workshops (ICSTW), 2014 IEEE Seventh International Conference on. IEEE, 302-311.

[4] Alexander Bachmutsky. 2011. System design for telecommunication gateways. John Wiley \& Sons.

[5] Deepika Badampudi and Claes Wohlin. 2016. Bayesian Synthesis for Knowledge Translation in Software Engineering: Method and Illustration. In Software Engineering and Advanced Applications (SEAA), 2016 42th Euromicro Conference on. IEEE, 148-156.

[6] Ishan Banerjee, Bao Nguyen, Vahid Garousi, and Atif Memon. 2013. Graphical user interface (GUI) testing: Systematic mapping and repository. Information and Software Technology 55, 10 (2013), 1679-1694.

[7] Stefan Berner, Roland Weber, and Rudolf K Keller. 2005. Observations and lessons learned from automated testing. In Software Engineering, 2005. ICSE 2005. Proceedings. 27th International Conference on. IEEE, 571-579.

[8] Virginia Braun and Victoria Clarke. 2006. Using thematic analysis in psychology. Qualitative research in psychology 3, 2 (2006), 77-101.

[9] Eliane Figueiredo Collins and Vicente Ferreira de Lucena. 2012. Software test automation practices in agile development environment: An industry experience report. In Proceedings of the 7th International Workshop on Automation of Software Test. IEEE Press, 57-63.

[10] G Mohan Doss Gandhi and Anitha S Pillai. 2014. Challenges in GUI Test Automation. International fournal of Computer Theory and Engineering 6, 2 (2014), 192.

[11] N. Garousi, V. Senthil Anand and R. Bhavani. 2012. Software test automation the ground realities realized. Theoretical and Applied Information Technology 43, 2 (2012), 306-312.

[12] Vahid Garousi and Frank Elberzhager. 2017. Test Automation: Not Just for Test Execution. IEEE Software 34, 2 (2017), 90-96.

[13] Vahid Garousi and Mika V Mäntylä. 2016. When and what to automate in software testing? A multi-vocal literature review. Information and Software Technology 76 (2016), 92-117

[14] Vahid Garousi and Dietmar Pfahl. 2016. When to automate software testing? A decision-support approach based on process simulation. Fournal of Software: Evolution and Process 28, 4 (2016), 272-285.

[15] Mark Grechanik, Qing Xie, and Chen Fu. 2009. Maintaining and evolving GUIdirected test scripts. In Proceedings of the 31st International Conference on Software Engineering. IEEE Computer Society, 408-418.

[16] Theodore Hellmann, Elham Moazzen, Abhishek Sharma, Md Zabedul Akbar, Jonathan Sillito, Frank Maurer, et al. 2014. An exploratory study of automated gui 
testing: Goals, issues, and best practices. Technical Report. University of Calgary.

[17] C Kaner. [n.d.]. Avoiding shelfware: a manager's view of automated GUI testing [online]. 1998. Dostupné $z$ : http://www. kaner. com/pdfs/autosqa. pdf [vid. 31. 3. 2012] ([n. d.]).

[18] Katja Karhu, Tiina Repo, Ossi Taipale, and Kari Smolander. 2009. Empirical observations on software testing automation. In Software Testing Verification and Validation, 2009. ICST'09. International Conference on. IEEE, 201-209.

[19] B. A. Kitchenham, T. Dyba, and M. Jorgensen. 2004. Evidence-based software engineering. In Proceedings. 26th International Conference on Software Engineering. 273-281. https://doi.org/10.1109/ICSE.2004.1317449

[20] Grischa Liebel, Emil Alégroth, and Robert Feldt. 2013. State-of-practice in GUIbased system and acceptance testing: An industrial multiple-case study. In Software Engineering and Advanced Applications (SEAA), 2013 39th EUROMICRO Conference on. IEEE, 17-24.

[21] Chang Liu. 2000. Platform-independent and tool-neutral test descriptions for automated software testing. In Proceedings of the 22nd international conference on Software engineering. ACM, 713-715.

[22] Atif M Memon, Martha E Pollack, and Mary Lou Soffa. 2001. Hierarchical GUI test case generation using automated planning. IEEE transactions on software engineering 27, 2 (2001), 144-155.

[23] Mehdi Mirzaaghaei, Fabrizio Pastore, and Mauro Pezze. 2010. Automatically repairing test cases for evolving method declarations. In Software Maintenance (ICSM), 2010 IEEE International Conference on. IEEE, 1-5.

[24] Donovan Lindsay Mulder and Grafton Whyte. 2013. A Theoretical Review of the Impact of Test Automation on Test Effectiveness. In Proceedings of The 4th International Conference on Information Systems Management and Evaluation ICIME 2013. 168.

[25] Sanjeev Patwa and Anil Kumar Malviya. 2010. Testability of software systems. International fournal of Research and Reviews in Applied Sciences 5, 1 (2010).

[26] Bret Pettichord. 2002. Design for testability. In Pacific Northwest Software Quality Conference. 1-28.

[27] Leandro Sales Pinto, Saurabh Sinha, and Alessandro Orso. 2012. Understanding myths and realities of test-suite evolution. In Proceedings of the ACM SIGSOFT 20th International Symposium on the Foundations of Software Engineering. ACM, 33.

[28] Dudekula Mohammad Rafi, Katam Reddy Kiran Moses, Kai Petersen, and Mika V Mäntylä. 2012. Benefits and limitations of automated software testing: Systematic literature review and practitioner survey. In Proceedings of the 7th International Workshop on Automation of Software Test. IEEE Press, 36-42.

[29] Colin Robson. 2002. Real world research: a resource for social scientists and practitioner. Adapting Open Innovation in ICT Ecosystem Dynamics References Real World Research: A Resource for Social Scientists and Practitioner (2002), 270.

[30] Colin Robson and Kieran McCartan. 2016. Real world research. John Wiley \& Sons.

[31] Anderson Rodrigues and Arilo Dias-Neto. 2016. Relevance and Impact of Critical Factors of Success in Software Test Automation lifecycle: A Survey. In Proceedings of the 1st Brazilian Symposium on Systematic and Automated Software Testing. ACM, 6.

[32] Anderson Rodrigues, Arilo C Dias-Neto, and Allan Bezerra. 2015. TAPN: Test Automation's Pyramid of Needs. In XIV Brazilian Symposium on Software Quality. SBQS, 122-136.

[33] Zahra Sahaf, Vahid Garousi, Dietmar Pfahl, Rob Irving, and Yasaman Amannejad. 2014. When to automate software testing? decision support based on system dynamics: an industrial case study. In Proceedings of the 2014 International Conference on Software and System Process. ACM, 149-158.

[34] Keith Stobie. 2009. Too much automation or not enough? When to automate testing. In Pacific Northwest Software Quality Conference.

[35] Ossi Taipale, Jussi Kasurinen, Katja Karhu, and Kari Smolander. 2011. Trade-off between automated and manual software testing. International fournal of System Assurance Engineering and Management 2, 2 (2011), 114-125.

[36] Laurie Williams, Gunnar Kudrjavets, and Nachiappan Nagappan. 2009. On the effectiveness of unit test automation at microsoft. In Software Reliability Engineering, 2009. ISSRE'09. 20th International Symposium on. IEEE, 81-89.

[37] Claes Wohlin. 2014. Guidelines for snowballing in systematic literature studies and a replication in software engineering. In Proceedings of the 18th international conference on evaluation and assessment in software engineering. ACM, 38.

[38] Qing Xie and Atif M Memon. 2007. Designing and comparing automated test oracles for GUI-based software applications. ACM Transactions on Software Engineering and Methodology (TOSEM) 16, 1 (2007), 4. 